\title{
Molecular iodine/doxorubicin neoadjuvant treatment impair invasive capacity and attenuate side effect in canine mammary cancer
}

Xóchitl Zambrano-Estrada ${ }^{1}$, Brianda Landaverde-Quiroz ${ }^{2}$, Andrés A. Dueñas-Bocanegra², Marco A. De Paz-Campos², Gerardo Hernández-Alberto², Benjamín Solorio-Perusquia ${ }^{3}$, Manuel Trejo-Mandujano ${ }^{3}$, Laura Pérez-Guerrero ${ }^{3}$,

Evangelina Delgado-González ${ }^{1}$, Brenda Anguiano ${ }^{1}$ and Carmen Aceves ${ }^{1 *}$ (D)

\begin{abstract}
Background: Mammary cancer has a high incidence in canines and is an excellent model of spontaneous carcinogenesis. Molecular iodine $\left(I_{2}\right)$ exerts antineoplastic effects on different cancer cells activating re-differentiation pathways. In co-administration with anthracyclines, $I_{2}$ impairs chemoresistance installation and prevents the severity of side effects generated by these antineoplastic drugs. This study is a random and double-blind protocol that analyzes the impact of $\mathrm{I}_{2}(10 \mathrm{mg} /$ day) in two administration schemes of Doxorubicin (DOX; $30 \mathrm{mg} / \mathrm{m} 2)$ in 27 canine patients with cancer of the mammary gland. The standard scheme (sDOX) includes four cycles of DOX administered intravenously for 20 min every 21 days, while the modified scheme (mDOX) consists of more frequent chemotherapy (four cycles every 15 days) with slow infusion (60 min). In both schemes, I 2 or placebo (colored water) was supplemented daily throughout the treatment.
\end{abstract}

Results: mDOX attenuated the severity of adverse events (VCOG-CTCAE) in comparison with the sDOX group. The overall tumor response rate (RECIST criteria) for all dogs was 18\% (interval of reduction 48-125\%), and no significant difference was found between groups. $I_{2}$ supplementation enhances the antineoplastic effect in mDOX, exhibiting a significant decrease in the tumor epithelial fraction, diminished expression of chemoresistance (MDR1 and Survivin) and invasion (UPA) markers and enhanced expression of the differentiation factor known as peroxisome proliferator-activated receptors type gamma (PPARY). Significant tumor lymphocytic infiltration was also observed in both $\mathrm{I}_{2}$-supplemented groups. The ten-month survival analysis showed that the entire $I_{2}$ supplementation (before and after surgery) induced $67-73 \%$ of disease-free survival, whereas supplementation in the last period (only after surgery) produced $50 \%$ in both schemes.

Conclusions: The $\mathrm{mDOX}+\mathrm{l}_{2}$ scheme improves the therapeutic outcome, diminishes the invasive capacity, attenuates the adverse events and increases disease-free survival. These data led us to propose $\mathrm{mDOX}+\mathrm{I}_{2}$ as an effective treatment for canine mammary cancer.

Keywords: Canine mammary cancer, Molecular iodine, Neoadjuvant chemotherapy, Doxorubicin, Animal welfare

\footnotetext{
* Correspondence: caracev@unam.mx

${ }^{1}$ Instituto de Neurobiología, Universidad Nacional Autónoma de México,

Boulevard Juriquilla 3001, CP 76230 Querétaro, Mexico

Full list of author information is available at the end of the article
} 


\section{Background}

Mammary cancer is the neoplasia with most incidences in intact female dogs [1]. Currently, surgery remains the mainstay of canine mammary cancer treatment, but several studies describe adjuvant chemotherapy as an approach that increases quality of life and disease-free survival [2-5]. In women, the use of systemic chemotherapy before local surgical excision, known as neoadjuvant therapy, may also reduce the extent of local surgery without jeopardizing patient survival and disease-free survival [6]. This practice is still scarce in dogs, but recent reports describe the use of this approach to transform unresectable tumors into resectable ones even though disease-free survival or survival time was not significantly longer when different chemotherapeutic drugs were used as monotherapy [7-9].

One of the most important considerations in chemotherapy treatment is the attenuation of toxicity and chemoresistant effects. Slow infusion is one of the most explored strategies to reduce toxicity. Doxorubicin (DOX), a widely used therapeutic drug, exhibited a direct correlation between its toxic effects and its peak plasma concentration. Rapid intravenous bolus results in higher peak levels, whereas slow infusion rates lead to a greater area under the curve associated with less toxicity. However, the pharmacokinetics of this drug could be different between species. In humans, slow infusion (1 to $6 \mathrm{~h}$ or more) exhibited less toxicity in cardiac tissue and other organs [10], whereas in rodents fast infusion (5 versus $30 \mathrm{~min}$ ) seems to be better protection [11]. Related studies in canines were not available until now. A second strategy, more represented in canine studies, is the use of chemotherapeutic drugs in different schemes or pharmaceutical presentations (metronomic therapy, liposomes, nanoparticles) [12-15], specific molecules that act as inhibitors of growth factor receptors (toceranib phosphate) $[16,17]$, components that exacerbate immune anti-tumor responses (anti Her2, TNF $\alpha$ ) [18, 19] or even natural products with adjuvant antineoplastic effects (Curcumin) [20].

In this regard, previous work by several research groups has shown that molecular iodine $\left(\mathrm{I}_{2}\right)$ exerts antineoplastic effects on different cancer cells grown under basal, chemoresistant and stem (spheroid) conditions by activating re-differentiation pathways and reversing chemoresistance and epithelial-mesenchymal transition (EMT) [21-23]. Similarly, pre-clinic (immunosuppressed mice and pharmacologically induced rats) and clinic mammary cancer protocols have shown that $I_{2}$ supplementation inhibits tumor growth, activates the antitumor immune system and exerts antioxidant actions that prevent the severity of side effects generated by antineoplastic drug toxicity $[24,25]$. The antineoplastic actions of $\mathrm{I}_{2}$ include direct mechanisms exerting oxidant effects on the mitochondrial membrane and indirectly generating an iodinated lipid known as 6-iodolactone (6IL), a specific ligand of peroxisome proliferator-activated receptor gamma (PPARY) [26]. PPARs are ligandactivated transcription factors, and three subtypes have been identified (PPAR alpha, beta, and gamma). PPARy is involved in lipid metabolism and has recently been shown to play a significant role in cell proliferation, differentiation, and apoptosis in many types of cancer [27]. The present study was designed to analyze the effect of $\mathrm{I}_{2}$ supplementation in two DOX administration schedules: standard (21-day cycles; 20 min infusion) versus modified (15-day cycles; $60 \mathrm{~min}$ infusion) regimens in canine patients with mammary cancer.

\section{Methods}

Patients

The study was performed to include at least between 4 and 10 dogs per group according to our previous studies in rodents [25]. We invited and registered 93 patients who gradually abandoned the protocol for various reasons. Some of them related to the side effects (although we were very attentive to their health and well-being, many owners did not accept the loss of hair or the temporary change of mood), or due to other difficulties (abandonment of treatment, death independent of cancer, pyometra, etc.). As the study was double-blind, the confirmation of the groups was revealed until the day of the surgery and it was not possible to re-adjust them. Twenty-seven intact female dogs with acceptable health and a diagnosis of mammary cancer finished the protocol. All procedures were performed at the Veterinary Hospital of the Universidad Autónoma de Querétaro and at the Veterinary Hospital of FES-Cuautitlán, UNAM. Additionally, all procedures followed the Animal Care and Use program (NIH, USA) and were approved by the Research Ethics Committee of INBUNAM (Protocol \#102).

\section{Clinical protocol}

Cytological diagnostic of mammary cancer was performed by a sample of puncture fine needle aspiration. Eligibility criteria also included signed informed consent and normal hepatic, renal and cardiovascular function (general blood and urine analysis and electrocardiogram, respectively). A biopsy was performed in accepted dogs to confirm the cytological diagnosis and determine the tumor classification according to Goldschmidt et al. [28]. A dog health questionnaire was filled out by the veterinary surgeon and the dog owner before every chemotherapy cycle. Toxicity was graded using the Veterinary Cooperative Oncology Group - Common Terminology Criteria for Adverse Events (VCOG-CTCAE) [29]. The clinical signs considered in the present work 
were weight, vomitus, diarrhea, anorexia, lethargy, anemia and neutropenia. Distant metastasis disease was identified using ultrasound and thorax radiographs.

\section{Chemotherapy}

The standard scheme (sDOX) comprises four cycles of DOX $\left(30 \mathrm{mg} / \mathrm{m}^{2}\right)$ administered intravenously for $20 \mathrm{~min}$ every 21 days, while the modified regimen (mDOX) consists in the same amount of chemotherapy infused for $60 \mathrm{~min}$ in four, 15-day cycles.

\section{Molecular iodine}

Was prepared with Lugol solution (Golden Bell, Edo. de Mexico, Mx) and diluted in distilled water. Iodine $(10 \mathrm{mg} /$ day) or placebo (water with artificial food coloring) was administered orally with daily food 1 week before chemotherapy and continuously until mastectomy. The file for each dog was opened on the day of the mastectomy to identify the subgroup ( $\mathrm{I}_{2}$ or placebo). To analyze the effect of $\mathrm{I}_{2}$ supplementation on survival and disease-free survival rates, all patients received the halogen supplementation for ten more months.

\section{Mastectomy}

The veterinary surgeon carried out the pre-surgical protocol and the corresponding postoperative monitoring during the peri-mastectomy period. The surgery was performed 6 weeks after the last chemotherapy to ensure the complete re-establishment of the patients' health. Anesthesia included premedication with $0.01 \mathrm{mg} / \mathrm{kg}$ Acepromazine (Vetoquinol, Mexico City, MX), induction and maintenance with $2 \mathrm{mg} / \mathrm{kg}$ Propofol (App pharmaceutics, Los Angeles, CA) and fluid therapy (saline solution). For pain management, $0.005-0.02 \mu \mathrm{g} / \mathrm{kg}$ with Buprenorphine (Schering-Plough, NJ, USA), $0.2 \mathrm{mg} / \mathrm{kg}$ Meloxicam (Aranda Lab, Queretaro, MX) and $1 \mathrm{mg} / \mathrm{kg}$ Tramadol (Pisa, Mexico City, MX), as well as antibiotic therapy with $5 \mathrm{mg} / \mathrm{kg}$ Enrofloxacin (Bayer, Edo de Mexico, MX).

\section{lodine consumption, thyroid status, cardiac damage and tumor response}

All these parameters were recorded on the day that the dogs were accepted into the protocol (initial) and on the day of the mastectomy (final). The content of total iodine in urine was determined by ionic analysis (potentiometer Thermo Scientific Orion Star A214 with ion selective electrode; ISE; LIS-146ICM), triiodothyronine (T3) and thyrotropin (TSH) circulating levels by ELISA (International immuno-diagnostics/IIDE-2021 and Abnova/KA2296, respectively). Serum creatine kinase type $\mathrm{MB}$ (CK-MB) activity, used as a marker of cardiac damage, was measured by using the CK-MB Test (Stanbio Laboratory/0980-103). Electrocardiogram (ECG) recordings were made over a minimum 30 -s period in each dog, while conscious and lightly restrained, on initial (time 0), $10 \mathrm{~min}$ before each chemotherapy session and 1 week before mastectomy. The ECG traces from each animal were examined by a certified veterinary cardiologist to determine the following variables: heart rate, P-wave duration, P-wave amplitude, PR interval, QRS duration, QRS amplitude, QT interval, ST segment structure, and T-wave amplitude.

The tumor response rate was determined according to the Response Evaluation Criteria in Solid Tumors (RECIST) which use only the longest diameters of tumors in the axial plane [30]. The response is placed into one of four categories: complete response (CR; disappearance of all target lesions), partial response (PR; $\geq$ $50 \%$ decrease in sum of target lesions from baseline), stable disease (SD; meets criteria for PR or PD) or progressive disease (PD; $\geq 25 \%$ increase in sum of target lesions or appearance of new lesions).

\section{Immunohistochemistry}

Was performed on 3 or $5 \mu \mathrm{m}$ formalin-fixed paraffinembedded specimens that were float-mounted onto silanized glass slides. ER $\alpha$ was analyzed by a sandwich protocol (polyclonal first antibody HC-20, Santa Cruz Biotechnology. Dallas, Texas, USA; and goat anti-rabbitimmunoglobulin, peroxidase labeled, Dako/K4011). Sections were counterstained with hematoxylin. ER $\alpha$ positive cells were identified by the presence of a brown stain over the nucleus and cytoplasm. Five regions were analyzed, and tumor samples were considered ER $\alpha$ positive when 20 or more cells per field were positively labeled in the nucleus, in the cytoplasm or in both, and were found in at least three fields at 40X. Negative controls were obtained by using only the secondary antibody (Dako/K4011). The positive control was performed employing ovary tissue.

\section{Histopathological analysis}

Classification and grading of mammary tumors was determined by Goldschmidt et al. criteria which include tubule formation, nuclear pleomorphism and mitoses/ hyperchromatism [28]. The histologic malignancy was described as well differentiated (low values), moderately differentiated (intermediate values) and poorly differentiated (high values). Hematoxylin and eosin (H\&E) staining was used to observe histological malignancy and lymphocytic infiltration. Masson's trichrome staining was used to identify epithelial (red) and connective (blue) proportions of representative slides per group. Two independent observers evaluated all these characteristics in anonymized and blinded samples. The analysis was performed as the mean of three random regions. The number of lymphocytes and the 
percentages of stained areas were calculated using the Image 1.47 program (Wayne Rasband. NIH, USA).

\section{Molecular expression}

Bax, Bcl2, urokinase plasminogen activator (uPA), Survivin, multidrug resistance protein 1 (MDR1) and PPAR expression were analyzed by quantitative real-time PCR (qPCR) [21]. Total RNA was obtained using the TRIZOL reagent (Life Technologies, Inc., Carlsbad, CA), and the extracted RNA $(2 \mu \mathrm{g})$ was reverse transcribed using oligo-deoxythymidine. qPCR was performed with SYBR green dye (Thermo Scientific/K0221) in detector system Roto-Gene 3000 (Corbett Research, Mortlake, NSW, Australia) with the canine gene-specific primers listed in Table 1. Validation of $\mathrm{qPCR}$ was performed using $\beta$ actin and phosphoglycerate kinase 1 (PGK1) as nonregulated housekeeping genes. Gene expression was calculated using the D cycle threshold method and normalized to $\beta$-actin content.

\section{Statistical analysis}

All statistical analysis was performed in Prism version 6.01 (GraphPad Software). An unpaired, Student's $t$-test was used to compare average (initial and final) of total urine iodine, serum thyroid hormones and cardiac enzyme (CK-MB). Unpaired one-way ANOVAs were used to analyze electrocardiogram profile, residual tumor size and epithelial/connective tissue proportion. The differential gene response was performed with Mann-Whitney $\mathrm{U}$, and Pearson correlation was used to determine correlations between residual tumor size and lymphocyte number. A $p$ value of less than 0.05 was considered significant.

\section{Results}

Table 2 summarizes the patients' characteristics. The median age was $9.2 \pm 2.4$ years, and the most affected breed was the Standard Poodle (33\%). Sixty-eight percent of dogs were nulliparous, and none received hormone therapy. Only 20\% (6 dogs) of the patients exhibited overweight (between 10\% and 30\% above standard weight). Two patients presented distant metastasis (lungs) at the time of diagnosis (clinical state V).

Grading of adverse events for each group throughout the treatment is summarized in Fig. 1. Patients started with excellent conditions (0), and lower and medium grades (1 to 2 ) were observed after the second or third chemotherapy cycle (before the third or fourth chemotherapy cycle, respectively). No patients exhibited significant weight loss or developed grades 3,4 or 5 (death) during treatments. Table 3 describes the number of patients who presented clinical signs and their severity (grade) at any time during chemotherapy treatment. The mDOX scheme reduces the severity of the general symptoms in comparison with sDOX. The $\mathrm{I}_{2}$ supplement further attenuates the severity of the symptoms in both treatments. The six dogs that presented grade 2 vomiting and/or diarrhea were complemented with supportive care: antibiotic (Metronidazole, $30-40 \mathrm{mg} / \mathrm{kg}$ for 14 days) and probiotics (Enterogermina 2 billion $/ 5 \mathrm{~mL}$ ); with Omeprazole $(20 \mathrm{mg} / \mathrm{kg}$ for $24 \mathrm{~h}$ ), Ranitidine $(3 \mathrm{mg} / \mathrm{kg}$ every $12 \mathrm{~h}$ for 8 days), Difenidol (1 $\mathrm{mg} / \mathrm{kg}$ for 4 days), and/or fluid therapy (saline solution).

$\mathrm{I}_{2}$ ingestion, thyroid status and cardiac damage were recorded when patients were accepted into the protocol (initial) and on the day of mastectomy (final). Total urinary iodine values were above $2000 \mu \mathrm{g} / \mathrm{L}$ in supplemented patients and DOX groups did not show any changes. $\mathrm{I}_{2}$ supplementation and/or DOX administration neither modified T3 or TSH circulating values or showed cardiac damage measured by serum CK-MB activity (Fig. 2). The electrocardiogram variables from initial (time 0) and final (before mastectomy) are summarized in Fig. 3. No significant changes were observed between groups in any variable or protocol day.

Figure 4 shows the tumor classification, histological grade and residual tumor size. Most patients developed more than one tumor (Table 2). The tumors with enough size (more than $1 \mathrm{~cm}^{3}$ ) were analyzed as individual tumors. Ninety-one percent were of the epithelial type, whereas only $9 \%$ corresponded to the mixed type (carcinosarcoma). Histological grading showed that $60 \%$

Table 1 Oligonucleotides

\begin{tabular}{|c|c|c|c|c|}
\hline Gen & Reference & Forward primer & Reverse primer & $\mathrm{pb}$ \\
\hline$\overline{\beta-\operatorname{actin}^{1}}$ & NM_001101 & acagagtacttgcgctcagga & ccatcatgaagtgtgacgttg & 175 \\
\hline$P G K 1^{2}$ & NM_053291.3 & tgactttggacaagctggacgtga & cagcagccttgatcctttggttgt & 110 \\
\hline $\operatorname{Bax}^{3}$ & NM_001003011.1 & aagctgagcgagtgtctcaagcgc & tcccgccacaaagatggtcacg & 366 \\
\hline $\mathrm{BCl}_{2}^{4}$ & NM_000633.2 & gtggaggagctcttcaggga & aggcacccagggtgatgcaa & 304 \\
\hline PPARY ${ }^{5}$ & XM_005632014.1 & ttccattctcaagagcggaccc & tctccacagactcggcattcaa & 190 \\
\hline Survivin ${ }^{6}$ & NM_001003348.1 & accgcgtctctacgttcaag & ccaagtctggctcgttctca & 114 \\
\hline $\mathrm{UPA}^{7}$ & XM_005618862.1 & ttggggagatgaagtttgaggtgg & cagaacggatcttcagcaaggc & 105 \\
\hline$M D R 1^{8}$ & NM_001003215.1 & tatcagcagcccacgtcatc & cagccactgctacctacgag & 214 \\
\hline
\end{tabular}


Table 2 Patient characteristics

\begin{tabular}{|c|c|c|c|c|c|c|}
\hline Breed & Age (years) & Body weight (kg) & Clinical stage $(T N M) \varphi$ & Number of tumors & Parturition & ERa \\
\hline \multicolumn{7}{|c|}{ Standard scheme (Dox) } \\
\hline Standard Poodle & 12 & 6 & III & 2 & 0 & Positive \\
\hline Maltese bichon & 9 & 9.9 & III & 7 & 2 & Positive \\
\hline Dachshund & 13 & 6.6 & III & 4 & 2 & Positive \\
\hline Maltese bichon & 7 & 6.6 & III & 5 & 0 & Positive \\
\hline \multicolumn{7}{|c|}{ Standard scheme $\left(\operatorname{Dox}+\mathrm{I}_{2}\right)$} \\
\hline Rottweiler & 8 & 38 & III & 1 & 2 & Positive \\
\hline Standard Poodle & 12 & 5.8 & $\|$ & 5 & 0 & Positive \\
\hline Standard Poodle & 10 & 3.8 & III & 5 & 0 & Positive \\
\hline Labrador Retriever & 10 & 30 & III & 7 & 0 & Positive \\
\hline Standard Poodle & 12 & 6.4 & V & 8 & 1 & Positive \\
\hline Standard Poodle & 9 & 7 & III & 9 & 1 & Positive \\
\hline \multicolumn{7}{|c|}{ Modified scheme (Dox) } \\
\hline Cocker Spaniel & 9 & 10.3 & $\|$ & 8 & 0 & Positive \\
\hline Mixed breed & 6 & 4.2 & $\|$ & 2 & 0 & Positive \\
\hline Cocker Spaniel & 8 & 13 & । & 6 & 3 & Positive \\
\hline Chihuahua & 5 & 2.2 & 1 & 3 & 0 & Positive \\
\hline German Shepherd & 7 & 30.1 & III & 7 & 0 & Positive \\
\hline Dalmatian & 6 & 23 & । & 1 & 0 & Positive \\
\hline \multicolumn{7}{|c|}{ Modified scheme $\left(D o x+I_{2}\right)$} \\
\hline Cocker Spaniel & 10 & 16.8 & III & 1 & 0 & Positive \\
\hline Mixed breed & 8 & 7.6 & । & 5 & 0 & Positive \\
\hline Standard Poodle & 11 & 4.2 & V & 7 & 0 & Positive \\
\hline Cocker Spaniel & 13 & 9.8 & III & 7 & 0 & Positive \\
\hline Fox terrier Toy & 7 & 2.4 & । & 5 & 0 & Positive \\
\hline Standard Poodle & 10 & 4.5 & III & 3 & 0 & Positive \\
\hline Cocker Spaniel & 13 & 17.4 & III & 7 & 3 & Positive \\
\hline Standard Poodle & 10 & 6 & $\|$ & 8 & 0 & Positive \\
\hline Standard Poodle & 9 & 4.7 & III & 6 & 4 & Positive \\
\hline Chihuahua & 5 & 3.8 & III & 5 & 0 & Positive \\
\hline Dachshund & 10 & 5.2 & III & 5 & 1 & Positive \\
\hline
\end{tabular}

$\phi \mathrm{T}$ - Primary tumor, $\mathrm{N}$ - Regional lymph nodes, M - Distant metastasis [28];

Bold numbers, overweight animals (10-30\% of standard weight)

of tumors were well differentiated, $37 \%$ were moderately differentiated and only one (3\%) was poorly differentiated. No dogs exhibited a complete response (CR). One patient from $\mathrm{sDOX}+\mathrm{I}_{2}$ showed partial response (PR) and one from the sDOX group exhibited progressive disease (increases $\geq 25 \%$ ). The rest of the tumors (94\%) were maintained as stable disease. The overall response rate for all dogs was $18.0 \%$, where the residual tumor size corresponds to $82.0 \%$ (interval 48-125\%). However, even though tumor response (residual size) did not show statistical differences between groups, $\mathrm{I}_{2}$ supplementation was accompanied by decreases in epithelial tissue in comparison with connective content (Fig. 5). This observation was significant in the $\mathrm{mDOX}+\mathrm{I}_{2}$ group, suggesting that $\mathrm{I}_{2}$ acts on all tumor types by increasing the antineoplastic effect of mDOX.

The molecular analyses (Fig. 6) show that $I_{2}$ treatment generates similar responses in apoptotic and invasive markers independent of the DOX administration scheme. The presence of $\mathrm{I}_{2}$ increases the apoptotic index $(\mathrm{Bax} / \mathrm{Bcl} 2)$ and impairs the induction of invasive or chemoresistant genes such as MDR1, uPA and Survivin. The combination of $\mathrm{mDOX}+\mathrm{I}_{2}$ synergies the expression of Bax and PPAR $\gamma$ receptors.

One interesting phenomenon was observed in lymphocytic infiltration. $\mathrm{I}_{2}$ supplementation increases the 
STANDARD SCHEME

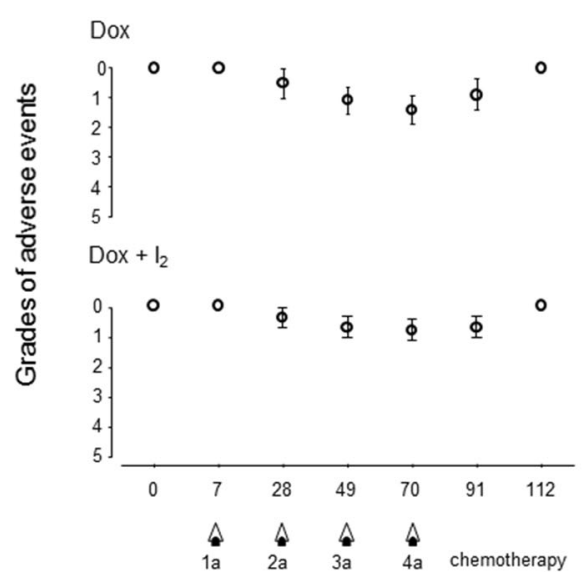

MODIFIED SCHEME

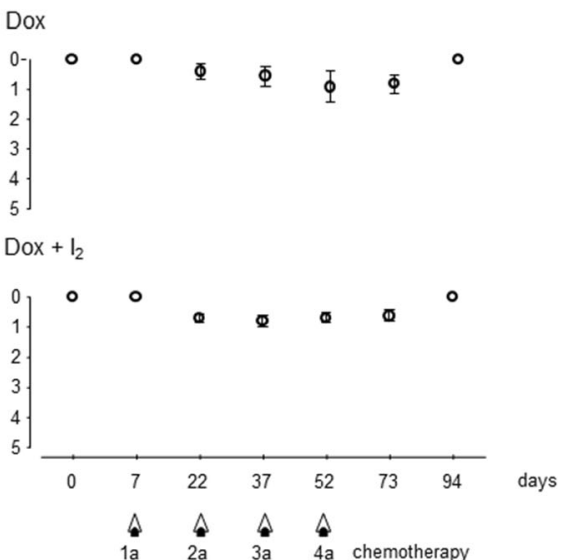

Fig. 1 Functional value according to the VCOG-CTCAE scale. Each point represents the mean and SD for each clinical report during all treatments. Arrows represent the day of chemotherapy (DOX) application

Table 3 Adverse events (VCOG-CTCAE)

\begin{tabular}{|c|c|c|c|c|c|}
\hline & Grade & sDox & $\mathrm{sDox}+\mathrm{I}_{2}$ & mDox & $\mathrm{mDox}+\mathrm{I}_{2}$ \\
\hline \multirow[t]{4}{*}{ Vomiting } & 1 & $1(25 \%)$ & $1(17 \%)$ & $1(17 \%)$ & $2(18 \%)$ \\
\hline & 2 & $1(25 \%)$ & $1(17 \%)$ & $1(17 \%)$ & \\
\hline & 3 & & & & \\
\hline & 4 & & & & \\
\hline \multirow[t]{4}{*}{ Diarrhea } & 1 & $1(25 \%)$ & $1(17 \%)$ & $1(17 \%)$ & $2(18 \%)$ \\
\hline & 2 & $1(25 \%)$ & $1(17 \%)$ & $1(17 \%)$ & $1(9 \%)$ \\
\hline & 3 & & & & \\
\hline & 4 & & & & \\
\hline \multirow[t]{4}{*}{ Anorexia } & 1 & $2(50 \%)$ & $1(17 \%)$ & $1(17 \%)$ & $1(9 \%)$ \\
\hline & 2 & $1(25 \%)$ & $1(17 \%)$ & & \\
\hline & 3 & & & & \\
\hline & 4 & & & & \\
\hline \multirow[t]{4}{*}{ Lethargy } & 1 & $1(25 \%)$ & 1 (17\%) & $1(17 \%)$ & $1(9 \%)$ \\
\hline & 2 & $1(25 \%)$ & $1(17 \%)$ & $1(17 \%)$ & $1(9 \%)$ \\
\hline & 3 & & & & \\
\hline & 4 & & & & \\
\hline \multirow[t]{4}{*}{ Anemia $^{a}$} & 1 & $2(50 \%)$ & $2(33 \%)$ & $2(33 \%)$ & $3(27 \%)$ \\
\hline & 2 & & & & \\
\hline & 3 & & & & \\
\hline & 4 & & & & \\
\hline \multirow[t]{4}{*}{ Neutropenia } & 1 & $1(25 \%)$ & $1(17 \%)$ & $1(17 \%)$ & $1(9 \%)$ \\
\hline & 2 & & & & \\
\hline & 3 & & & & \\
\hline & 4 & & & & \\
\hline
\end{tabular}

${ }^{a}$ Anemia includes: hematocrit, hemoglobin and erythrocyte values (mean globular volume and mean hemoglobin concentration) presence of lymphocytes in both schemes (Fig. 7) and exhibits a significant inverse correlation with the tumor response (more lymphocytes in the small residual tumor size).

As mentioned before, the use of DOX as monotherapy has the benefit of converting the unresectable tumors into resectable ones, but it does not increase the disease-free survival ( 207 days). With this premise, all our patients received $\mathrm{I}_{2}$ supplementation after the mastectomy, and the survival analysis was performed for ten more months ( $\sim 300$ days). Table 4 shows that $\mathrm{I}_{2}$ supplementation during the entire treatment (before and after surgery) has an improved disease-free survival of 67 and $73 \%\left(\mathrm{sDOX}+\mathrm{I}_{2}: 4\right.$ dogs and $\mathrm{mDOX}+\mathrm{I}_{2}: 8$ dogs, respectively) compared with the 50\% (sDOX: 2 dogs; mDOX: 3 dogs) observed in those that received $\mathrm{I}_{2}$ only in the last period (after chemotherapy). The two patients with previous metastasis (lungs) received $\mathrm{sDOX}+\mathrm{I}_{2}$ and $\mathrm{mDOX}$ $+\mathrm{I}_{2}$, each. In the first case, metastasis disappeared and after 10 months the patient was still alive without relapse or distant metastasis. In the second case (mDOX $+\mathrm{I}_{2}$ ), the metastasis progressed, and the patient died 4 months after surgery. Two patients (in the sDOX $+\mathrm{I}_{2}$ group) exhibited local mammary cancer relapse 7 months after surgery. Tumors were removed in a second surgery and after 10 months, both were still alive without evident metastasis. Three dogs died from causes independent of mammary cancer (two due to other cancers and one due to pyometra complications).

\section{Discussion}

In the present work, we compared two DOX administration schemes and the adjuvant effect of $I_{2}$ in canine mammary cancer. Our results showed that both DOX schemes are well tolerated, since no patients exhibited 


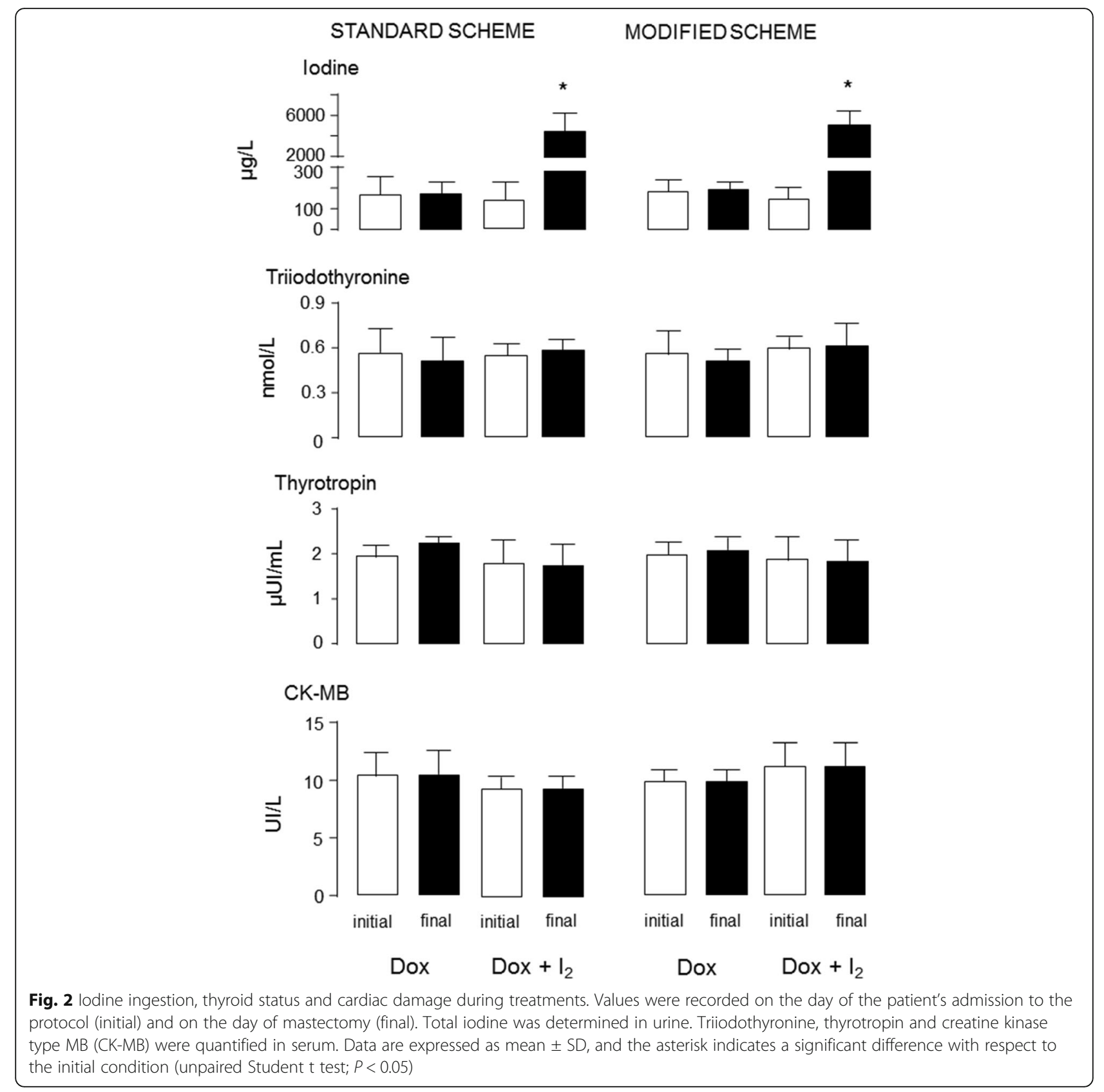

grades 3 or 4, according to the VCOG-CTCAE [29], in both conditions. Moreover, cardiac damage was not evident through serum CK-MB activity or ECG in any group. The clear attenuation of adverse events observed in $\mathrm{mDOX}$ agrees with previous reports in humans where slow infusion ( 1 to $6 \mathrm{~h}$ ) exhibited less toxicity [10], suggesting that the pharmacokinetics of DOX could be similar between humans and dogs. The addition of the $\mathrm{I}_{2}$ supplement appears to further attenuate the severity of adverse events. This benefit could be explained by the antioxidant effect of this chemical form of iodine, which is ten times more efficient than ascorbic acid and 100 times more potent than KI (Ferric reducing/antioxidant power assay; FRAP) in vitro [24]. Indeed, previous reports from our group in a rodent mammary cancer model showed that $\mathrm{I}_{2}$ supplementation prevented weight loss and cardiotoxicity (cardiac lipoperoxidation and serum CK-MB) secondary to DOX, without impairment of its synergistic antitumor action [24].

Regarding the tumor response (RECIST), the overall response was $18 \%$ showing that $94 \%$ of tumors were maintained as stable disease. This percentage of response is like that described by other authors when chemotherapy drugs are used in the form of 


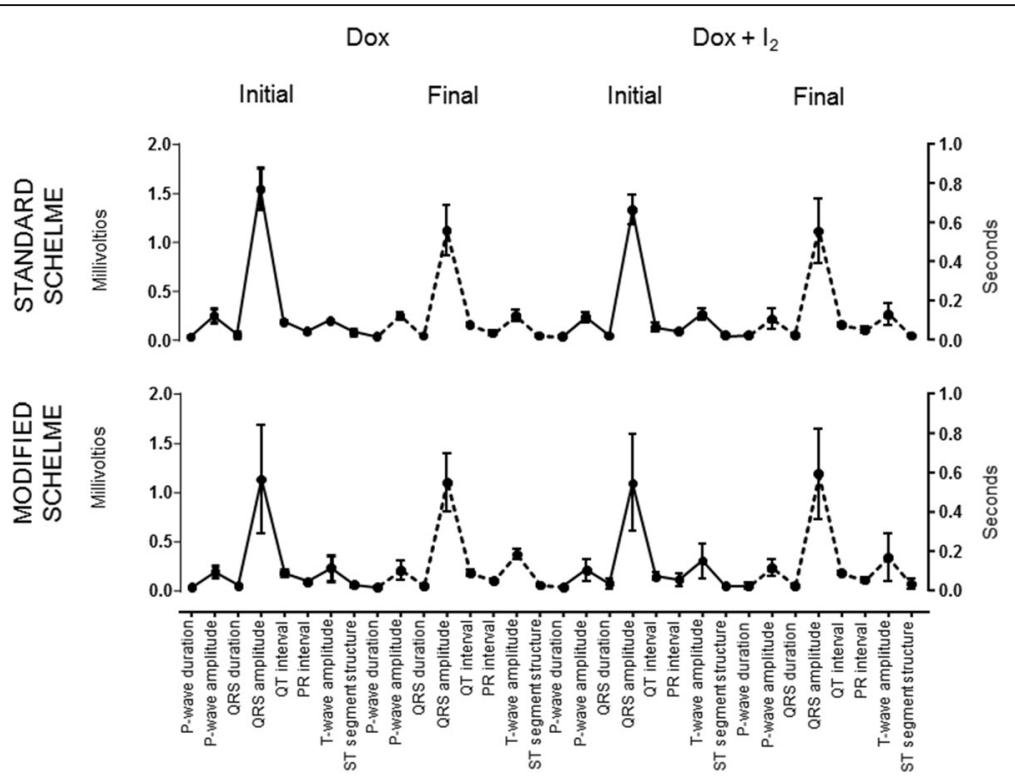

Fig. 3 Electrocardiogram profile. Values represent the day of the patient's admission to the protocol (initial) and 1 week before mastectomy (final). One-way ANOVA was performed for each variable and no significant differences were observed

monotherapy [6-8]; however, the important advantage observed in the $\mathrm{I}_{2}$-supplemented groups was the significant attenuation of invasive potential in the residual tumor mass. The tumor content showed a significant decrease in epithelial tissue, which is considered the source of tumor progression and metastasis, and a minimum expression of chemoresistance (MDR1 and Survivin) and invasion (uPA) markers, suggesting a better prognosis. It is well established that fibroblast and connective tissue are not $\mathrm{I}_{2}$-capturing tissues, whereas several types of epithelium uptake this halogen [31]. Moreover, it is described that cancerous cells exhibited between 10 and 100 times more sensitivity to apoptotic $I_{2}$ effects than its normal counterpart $[22,31]$. Two main pathways have been proposed to explain these effects; one concerning the direct oxidant/antioxidant action of $\mathrm{I}_{2}$ and a second pathway through iodolipid formation. Upadhyay et al. [32] showed that mitochondria isolated from the tumor (TT) and extra-tumoral tissue (ET) of human breast display significant uptake of iodine; but only TT mitochondria respond by increasing their permeability transition and releasing apoptogenic proteins, indicating a direct and differential proapoptotic action of $\mathrm{I}_{2}$ in mitochondria from cancerous cells. The second pathway is the generation of an iodinated derivative of arachidonic acid (AA), called 6-iodolactone (6-IL). AA is an essential membrane lipid, and its elevated concentrations in cancerous cells have been implicated in tumoral processes [33, 34]. 6-IL has been detected in tumor mammary glands from rats supplemented with $I_{2}$ [35], and this

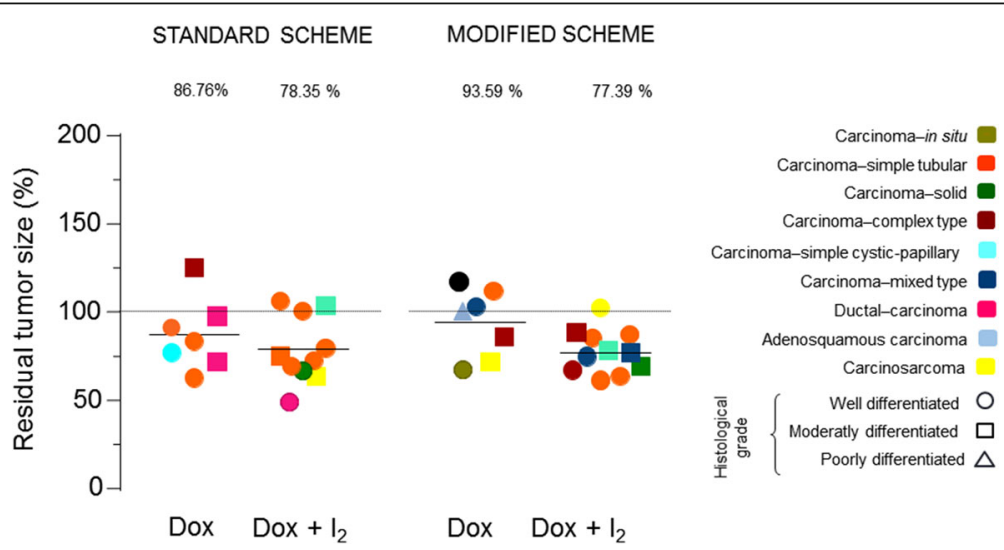

Fig. 4 Residual tumor size (\%), histopathological classification and grade of malignity were analyzed by RECIST scale. Each point represents an individual tumor. One-way ANOVA was performed, and no significant differences were observed 

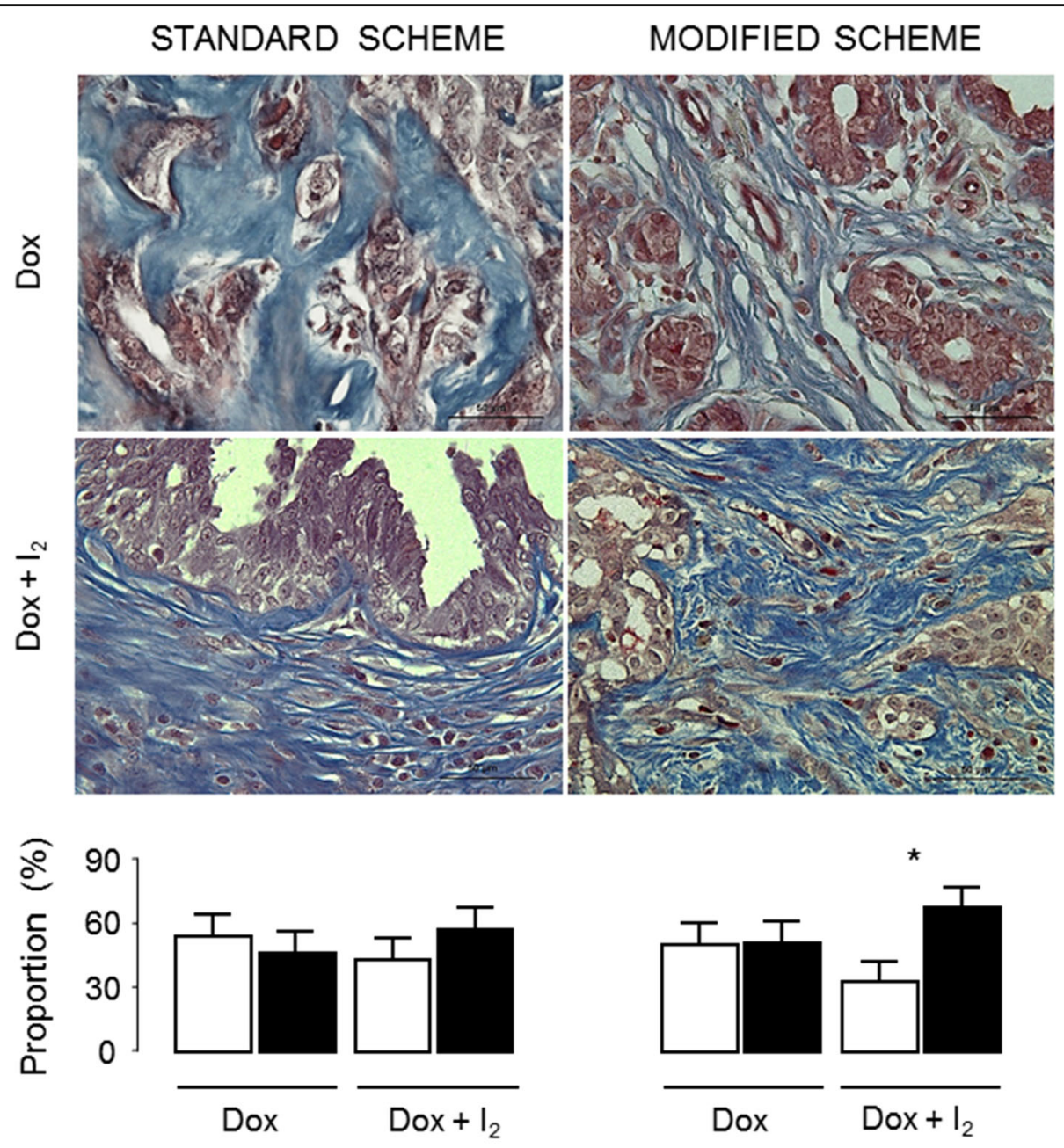

Epithelium

Connective tissue

Fig. 5 Epithelial and connective tissue proportion (\%) in the final tumor mass. Micrograph staining with Masson's trichrome method (red, epithelium; blue, connective tissue). Quantitative analysis was performed as the average of three random regions (40X) using the ImageJ 1.47 program. Data are expressed as mean $\pm \mathrm{SD}$, and the asterisk indicates a significant difference between groups. Student's $t$-test $(P<0.05)$

iodolipid is a specific agonist of PPAR $\gamma$ [36]. Treatment with PPAR $\gamma$ agonists inhibits cancer cell growth by inducing $\mathrm{G}_{0}-\mathrm{G}_{1}$ cell-cycle detention, promoting differentiation and reverting the EMT [37]. EMT is characterized by the suppression of adhesion molecule expression (e.g., E-cadherin), the induction of mesenchymal proteins such as $\mathrm{N}$-cadherin or Vimentin and the acquisition of chemoresistance by up-regulation of ATPbinding cassette $(\mathrm{ABC})$ transporters (such as MDR1) and anti-apoptotic markers like Bcl2, Bcl-xl or Survivin [38]. Our observation that $I_{2}$ supplementation in both schemes diminishes part of these markers (MDR1, Bcl2, uPA and Survivin) and increases the expression of PPAR $\gamma$ agrees with the notion that $\mathrm{I}_{2}$ exerts its antineoplastic effects through PPAR $\gamma$ activation. Moreover, the loss of invasive capacity of remnant cancerous tissue could explain the low rate of recurrence and metastasis found in our patients even after 10 months.

Another important finding was the significant inverse correlation of lymphocytic infiltration with the tumor response (residual tumor size) in samples from dogs treated with $I_{2}$ supplementation. Previous reports described that the presence of lymphocytic infiltration in breast cancer tissue predicts a positive response of neoadjuvant chemotherapy, and led to speculate that the inclusion of conventional chemotherapy with sensitization of the immune system could be a promising paradigm $[39,40]$. The specific mechanism of $\mathrm{I}_{2}$ in this putative immune modulation has not been elucidated, but it is demonstrated that several immune cell types can internalize $\mathrm{I}_{2}$, which can act as an anti- or proinflammatory agent depending on the cellular context 


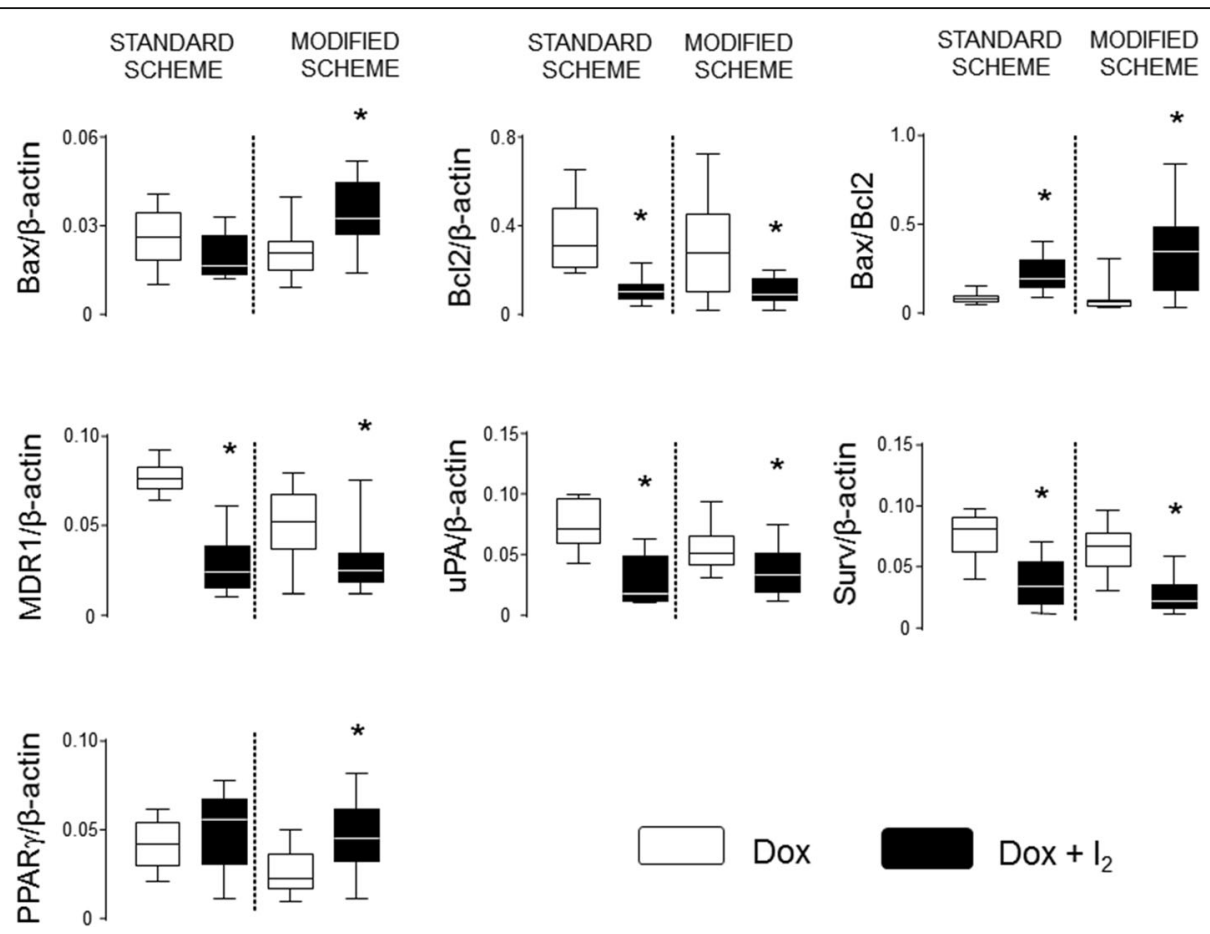

Fig. 6 Effect of treatments on the expression of chemoresistant, invasive and differentiation markers. RT-qPCR amplification were performed in residual tumors. Bax/Bcl2 index as apoptotic induction. Multidrug resistance protein 1 (MDR1); urokinase plasminogen activator (UPA), Survivin protein (Surv). Peroxisome proliferator-activated receptors type gamma (PPARY). Gene expression was calculated using the D cycle threshold method and normalized to $\beta$-actin content. Data are expressed as median and the asterisks indicate significant differences between DOX and $\mathrm{DOX}+\mathrm{I}_{2}$ groups in each treatment (Mann-Whitney $U ; P<0.05$ )

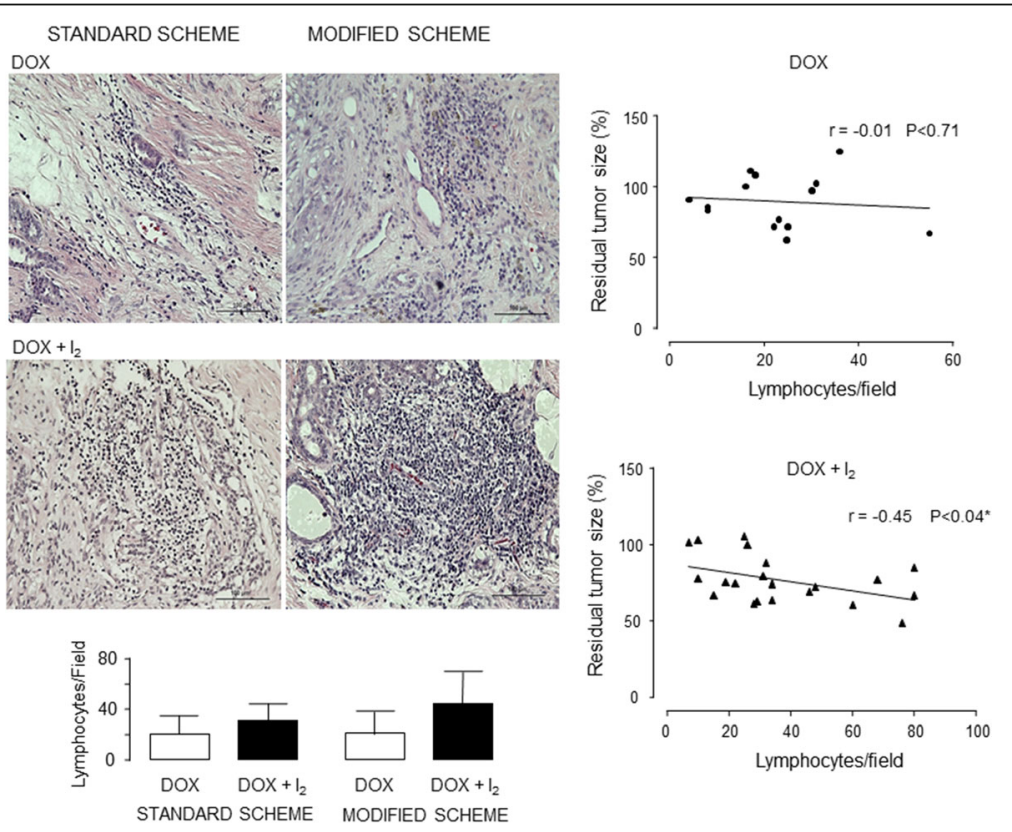

Fig. 7 Effect of treatments on lymphocytic infiltration. Micrographs stained with H\&E (20X). Quantitative analysis was performed as the average of three random regions using the ImageJ 1.47 program. Linear regression between residual tumor size (\%) and lymphocyte number from DOX and DOX $+\mathrm{I}_{2}$ groups (* Pearson coefficient, $p>0.04$ ) 
Table 4 Survival analysis

\begin{tabular}{|c|c|c|c|c|}
\hline Breed & Age (years) & Clinical stage (TNM) & Survival at ten months & Observations \\
\hline \multicolumn{5}{|c|}{ Standard scheme (Dox) } \\
\hline Standard Poodle & 12 & III & Yes & Alive without relapse \\
\hline Maltese bichon & 9 & III & No & Death, surgical complications \\
\hline Dachshund & 13 & III & Yes & Alive without relapse \\
\hline Maltese bichon & 7 & III & No & Death, surgical complications \\
\hline \multicolumn{5}{|c|}{ Standard scheme $\left(\operatorname{Dox}+\mathrm{I}_{2}\right)$} \\
\hline Rottweiler & 8 & III & Yes & Alive with relapse; no metastasis \\
\hline Standard Poodle & 12 & $\|$ & Yes & Alive without relapse \\
\hline Standard Poodle & 10 & III & Yes & Alive with relapse; no metastasis \\
\hline Labrador Retriever & 10 & III & Yes & Alive without relapse \\
\hline Standard Poodle & 12 & V & Yes & Alive without relapse \\
\hline Standard Poodle & 9 & III & Yes & Alive without relapse \\
\hline \multicolumn{5}{|c|}{ Modified scheme (Dox) } \\
\hline Cocker Spaniel & 9 & $\|$ & No & Death, transitional cell cancer \\
\hline Mixed breed & 6 & $\|$ & No & Euthanasia, suspected metastasis \\
\hline Cocker Spaniel & 8 & I & Yes & Alive without relapse \\
\hline Chihuahua & 5 & । & Yes & Alive without relapse \\
\hline German Shepherd & 7 & III & No & Death, anaplastic cancer \\
\hline Dalmatian & 6 & । & Yes & Alive without relapse \\
\hline \multicolumn{5}{|c|}{ Modified scheme $\left(\operatorname{Dox}+\mathrm{I}_{2}\right)$} \\
\hline Cocker Spaniel & 10 & III & Yes & Alive without relapse \\
\hline Mixed breed & 8 & I & Yes & Alive without relapse \\
\hline Standard Poodle & 11 & V & No & Death, extensive metastasis \\
\hline Cocker Spaniel & 13 & III & Yes & Alive without relapse \\
\hline Fox terrier Toy & 7 & । & Yes & Alive without relapse \\
\hline Standard Poodle & 10 & III & Yes & Alive without relapse \\
\hline Cocker Spaniel & 13 & III & No & Death, pyometra \\
\hline Standard Poodle & 10 & $\|$ & Yes & Alive without relapse \\
\hline Standard Poodle & 9 & III & Yes & Alive without relapse \\
\hline Chihuahua & 5 & III & Yes & Alive without relapse \\
\hline Dachshund & 10 & III & No & Death, surgical complications \\
\hline
\end{tabular}

$[41,42] . I_{2}$ can have anti-inflammatory effects either by suppressing the production of toxic oxygen intermediates in polymorphonuclear cells or by inhibiting neutrophil chemotaxis $[43,44]$. In contrast, $I_{2}$ may act directly on immune cells by inducing their reactivation. In chronic wounds, its presence activates the influx of macrophages and T cells [45]. In vitro, $\mathrm{I}_{2}$ enhances TNF $\alpha$ secretion from macrophages stimulated with bacterial lipopolysaccharides [46]. We observed a similar antitumor immune response in mammary cancer xenografts when mice Fox1 nu/nu were supplied with $\mathrm{I}_{2}$ (data not published). Moreover, PPAR $\gamma$ activation could be associated with such effects. In a recent publication, it was demonstrated that PPAR $\gamma$ expression increased almost 5-fold in a metronomic cyclophosphamide regime, suggesting that these receptors substantially contribute to the immune responsiveness of this treatment [47].

Finally, we wanted to test the effect of $I_{2}$ supplementation on disease-free-survival and survival when DOX is applied as monotherapy. There are only three previous reports that analyze this parameter using neoadjuvant chemotherapy in canines [6-8]. They found that survival time could be significantly extended with combination therapies (two or three drugs plus radiotherapy), rising the disease-free survival mean to 12 months (interval 213-521 days). In contrast, the disease-free survival only lasts 7 months (217 days) when DOX is used as monotherapy [7]. Our study included a ten-month ( 300 days) 
analysis, which showed that during this time overall disease-free survival was of $62 \% . \mathrm{I}_{2}$ increases the patient's survival when it is supplied from the beginning of treatment $\left(67 \%\right.$ in $\mathrm{sDOX}+\mathrm{I}_{2}$ and $73 \%$ in $\left.\mathrm{mDOX}+\mathrm{I}_{2}\right)$, and by $50 \%$ in both treatments when it is provided only after surgery. In the first case, $\mathrm{I}_{2}$ could act on two levels; first, by increasing the antineoplastic effects of DOX at the tumor site (differentiation induction, though less invasive phenotype and/or activation of immune response) and second, as an antioxidant, diminishing the oxidative damage in normal tissues (digestive, anemia, etc.) caused by DOX. The benefit after surgery (impaired relapse) could be linked to the antiproliferative effect of $\mathrm{I}_{2}$ per se. Several studies have reported that this antiproliferative action of $\mathrm{I}_{2}$ in mammary or prostatic pathologies (hyperplasia, mammary gland fibrosis or cancer) is useful only if the $I_{2}$ supplement is maintained at moderated high concentrations, whereas its suppression resumes pathology progression [48]. These effects agree with epidemiological studies showing that Japanese population that decreased $\mathrm{I}_{2}$ consumption increased the incidences in mammary and prostatic pathologies [49].

\section{Conclusions}

The combination of $\mathrm{DOX}+\mathrm{I}_{2}$ in the modified scheme improved the therapeutic outcome of mammary cancer, increasing both survival rate and relapse-free survival.

\section{Abbreviations \\ 6-IL: 6-iodolactone; ANOVA: Analysis of variance; DOX: Doxorrubicin; ECG: Electrocardiogram; EMT: Epithelial-mesenchymal transition; H\&E: Hematoxylin and eosin; $1_{2}$ : Molecular iodine; mDOX: Modified scheme; MDR1: Multidrug resistance protein 1; PPARY: Peroxisome proliferator- activated receptors type gamma; qPCR: Quantitative real-time PCR; RECIST: Response evaluation criteria in solid tumors; sDOX: Standard scheme; Surv: Survivin; T3: Triiodothyronine; TSH: Thyrotropin; uPA: Urokinase plasminogen activator; VCOG-CTCAE: Veterinary co-operative oncology group - common terminology criteria for adverse events \\ Acknowledgements \\ The authors appreciate the invaluable contribution of the Small Animal Veterinary Hospital of the Universidad Autónoma de Querétaro and Facultad de Estudios Superiores Cuautitlán-UNAM, proprietors of the patients, veterinary doctors and students. We also thank Alexander Bontempo, Maarten C. Antheny Werdler, Elsa Nydia Hernández Ríos, Brenda Ugalde Villanueva and María Juana Cárdenas for technical assistance; Lourdes Lara for academic support; Alberto Lara, Omar Gonzalez, Ramon Martínez and Sandra Hernández for computer assistance and Jessica González Norris for proofreading.}

\section{Ethical approval and consent to participate}

Protocol and clinical methods were accepted by the signed informed consent of the proprietors of the patients. Additionally, all procedures followed the Animal care and use program (NIH, USA) and were approved by the Research ethics committee of El Instituto de Neurobiología, Campus UNAM-Juriquilla (Protocol \#102).

\section{Funding}

This work was supported by grants from Programa de Apoyo a Proyectos de Investigación e Innovación Tecnológica (PAPIIT-UNAM; 200813, 209717 and 201516) and Consejo Nacional de Ciencia y Tecnología (235961). Xóchitl Zambrano-Estrada was a graduate student from the Programa de Doctorado en Ciencias de la Producción y de la Salud Animal at UNAM and received fellowship from CONACYT (356685). The funders had no role in study design, data collection and analysis, decision to publish, or preparation of the manuscript.

\section{Availability of data and materials}

The datasets used and/or analyzed during the current study are available from the corresponding author on reasonable request.

\section{Authors' contributions}

XZE performed the clinical protocols as the coordinator of both Veterinary Hospital teams, participated in scientific discussions and wrote the manuscript. BLQ, AADB, MADPC, and GHA performed the staff of Veterinary Hospital FES-Cuautitlan UNAM and were responsible for the care and management of canine patients; BSP, MTM, and LPG performed the faculty of Veterinary Hospital at UAQ and were responsible for the care and management of canine patients. EDG performed qPCR and thyroid status assay. BA contributed to statistical and scientific discussions. CA provided the concept design and academic direction and contributed to editing and drafting of the manuscript. All authors have read and approved the final document.

\section{Consent for publication}

Not applicable.

\section{Competing interests}

The authors declare that they have no competing interests.

\section{Publisher's Note}

Springer Nature remains neutral with regard to jurisdictional claims in published maps and institutional affiliations.

\section{Author details}

${ }^{1}$ Instituto de Neurobiología, Universidad Nacional Autónoma de México, Boulevard Juriquilla 3001, CP 76230 Querétaro, Mexico. ${ }^{2}$ Facultad de Estudios Superiores Cuautitlán, Universidad Nacional Autónoma de México, Mexico City, Mexico. ${ }^{3}$ Facultad de Ciencias Naturales, Universidad Autónoma de Querétaro, Mexico City, Mexico.

Received: 8 November 2017 Accepted: 5 March 2018

Published online: 12 March 2018

\section{References}

1. Pinho SS, Carvalho S, Cabral J, Reis CA, Gärtner F. Canine tumors: a spontaneous animal model of human carcinogenesis. Transl Res. 2012; 159:165-72.

2. Karayannopoulou M, Kaldrymidou E, Constantinidis TC, Dessiris A. Adjuvant post-operative chemotherapy in bitches with mammary cancer. J Vet Med A Physiol Pathol Clin Med. 2001;48:85-96.

3. Lavalle GE, De Campos CB, Bertagnolli AC, Cassali GD. Canine malignant mammary gland neoplasm with advanced clinical staging treated with carboplatin and cyclooxygenase innibitors. In Vivo. 2012:26:375-9.

4. Arenas C, Peña L, Granados-Soler JL, Pérez-Alenza MD. Adjuvant therapy for highly malignant canine mammary tumours: Cox-2 inhibitor versus chemotherapy: a case-control prospective study. Vet Rec. 2016; https://doi.org/10.1136/vr.103398.

5. Karayannopoulou M, Lafioniatis S. Recent advances on canine mammary cancer chemotherapy : a review of studies from 2000 to date. Revue Med Vet. 2016;167:192-200

6. Mauri D, Pavlidis N, loannidis JP. Neoadjuvant versus adjuvant systemic treatment in breast cancer: a meta-analysis. J Ntl Cancer Inst. 2005;97:188-94.

7. Wiley JL, Rook KA, Clifford CA, Gregor TP, Sorenmo KU. Efficacy of doxorubicin-based chemotherapy for non-resectable canine subcutaneous haemangiosarcoma. Vet Comp Oncol. 2010;8:221-33.

8. Mestrinho LA, Bernardo E, Niza MM, Lloret A, Buracco P. Neoadjuvant chemoradiotherapy and surgery as treatment for oral maxillary squamous cell carcinoma in a dog. Aust Vet J. 2012;90:264-8.

9. Wouda RM, Hocker SE, Higginbotham ML. Safety evaluation of combination carboplatin and toceranib phosphate (palladia) in tumour-bearing dogs: a phase I dose finding study. Vet Comp Oncol. 2017; https://doi.org/10.1111/vco.12332.

10. van Dalen EC van der Pal HJ. Kremer LC. Different dosage schedules for reducing cardiotoxicity in people with cancer receiving anthracycline 
chemotherapy. Cochrane Database Syst Rev. 2016:CD005008. https://doi.org/10.1002/14651858.

11. Tien CC, Peng YC, Yang FL, Subeq YM, Lee RP. Slow infusion rate of doxorubicin induces higher pro-inflammatory cytokine production. Regul Toxicol Pharmacol. 2016;81:69-76.

12. Polton G. Novel drug approaches in veterinary cancer therapy. Vet Ireland J. 2014;4:27-32.

13. Leach TN, Childress MO, Greene SN, Mohamed AS, Moore GE, Schrempp DR, Lahrman SR, Knapp DW. Prospective trial of metronomic chlorambucil chemotherapy in dogs with naturally occurring cancer. Vet Comp Oncol. 2012:10:102-12

14. Elmslie RE, Glawe P, Dow SW. Metronomic therapy with cyclophosphamide and piroxicam effectively delays tumor recurrence in dogs with incompletely resected soft tissue sarcomas. J Vet Intern Med. 2008;22:1373-9.

15. Vail DM, von Euler H, Rusk AW, Barber L, Clifford C, Elmslie R, Fulton L, Hirschberger J, Klein M, London C, Martano M, McNiel EA, Morris JS, Northrup N, Phillips B, Polton G, Post G, Rosenberg M, Ruslander D, Sahora A, Siegel S, Thamm D, Westberg S, Winter J, Khanna C. A randomized trial investigating the efficacy and safety of water soluble micellar paclitaxel (Paccal vet) for treatment of nonresectable grade 2 or 3 mast cell tumors in dogs. J Vet Intern Med. 2012;26:598-607.

16. London CA, Malpas PB, Wood-Follis SL, Boucher JF, Rusk AW, Rosenberg MP, Henry CJ, Mitchener KL, Klein MK, Hintermeister JG, Bergman PJ, Couto GC, Mauldin GN, Michels GM. Multi-center, placebo-controlled, double-blind, randomized study of oral toceranib phosphate (SU11654), a receptor tyrosine kinase inhibitor, for the treatment of dogs with recurrent (either local or distant) mast cell tumor following surgical excision. Clin Cancer Res. 2009;15:3856-65.

17. London C, Mathie T, Stingle N, Clifford C, Haney S, Klein MK, Beaver L, Vickery K, Vail DM, Hershey B, Ettinger S, Vaughan A, Alvarez F, Hillman L, Kiselow M, Thamm D, Higginbotham ML, Gauthier M, Krick E, Phillips B, Ladue T, Jones P, Bryan J, Gill V, Novasad A, Fulton L, Carreras J, McNeill C, Henry C, Gillings $S$. Preliminary evidence for biologic activity of toceranib phosphate (palladia ๑) in solid tumours. Vet Comp Oncol. 2012:10:194-205.

18. Fazekas J, Fürdös I, Singer J, Jensen-Jarolim E. Why man's best friend, the dog, could also benefit from an anti-HER-2 vaccine. Oncol Lett. 2016;12:2271-6.

19. Thamm DH, Kurzman ID, Clark MA, Ehrhart EJ, Kraft SL, Gustafson DL, Vail DM. Preclinical investigation of PEGylated tumor necrosis factor a in dogs with spontaneous tumors: phase I evaluation. Clin Cancer Res. 2010;16:1498-508.

20. Bolger GT, Licollari A, Tan A, Greil R, Vcelar B, Majeed M, Helson L. Distribution and metabolism of lipocurc ${ }^{T M}$ (liposomal curcumin) in dog and human blood cells: species selectivity and pharmacokinetic relevance. Anticancer Res. 2017;37:3483-92.

21. Aceves C, Anguiano B, Delgado G. The extrathyronine actions of iodine as antioxidant, apoptotic, and differentiation factor in various tissues. Thyroid. 2013;23:938-46.

22. Rösner H, Möller W, Groebner S, Torremante P. Antiproliferative/cytotoxic effects of molecular iodine, povidone-iodine and Lugol's solution in different human carcinoma cell lines. Oncol Lett. 2016;12:2159-62.

23. Bontempo A, Ugalde-Villanueva B, Delgado-González E, Rodríguez AL, Aceves $C$. Molecular iodine impairs chemoresistance mechanisms, enhances doxorubicin retention and induces downregulation of the CD44+/CD24+ and E-cadherin+/vimentin+ subpopulations in MCF-7 cells resistant to low doses of doxorubicin. Oncol Rep. 2017; https://doi.org/10.3892/or.2017.5934.

24. Alfaro Y, Delgado G, Cárabez A, Anguiano B, Aceves C. lodine and doxorubicin, a good combination for mammary cancer treatment antineoplastic adjuvancy, chemoresistance inhibition, and cardioprotection. Mol Cancer. 2013;12:45-56

25. Aceves C, Peralta G, Torres J, Delgado G, Domínguez A, De Obaldía R, Duarte LF, Paredes E, Avecilla C. lodine-supplemented diets prevents the development of resistance in breast cancer chemotherapy: participation of proliferative peroxisome-activated receptor gamma (PPARg). In: AACR special conference: Advances in Breast Cancer Research; 2011.

26. Nava-Villalba M, Nuñez-Anita RE, Bontempo A, Aceves C. Activation of peroxisome proliferator-activated receptor gamma is crucial for antitumoral effects of 6-iodolactone. Mol Cancer. 2015;14:168-72.

27. Yousefi B, Zarghami N, Samadi N, Majidinia M. Peroxisome proliferatoractivated receptors and their ligands in cancer drug- resistance: opportunity or challenge. Anti Cancer Agents Med Chem. 2016;16:1541-8.
28. Goldschmidt M, Peña L, Rasotto R, Zappulli V. Classification and grading of canine mammary tumors. Vet Pathol. 2011;48:117-31.

29. Veterinary cooperative oncology group - common terminology criteria for adverse events (VCOG-CTCAE) following chemotherapy or biological antineoplastic therapy in dogs and cats v1.1. Vet Comp Oncol. 2016;14:417-46.

30. Eisenhauer EA, Therasse P, Bogaerts J, Schwartz LH, Sargent D, Ford R, Dancey J, Arbuck S, Gwyther S, Mooney M, Rubinstein L, Shankar L, Dodd L, Kaplan R, Lacombe D, Verweij J. New response evaluation criteria in solid tumours: revised RECIST guideline (version 1.1). Eur J Cancer. 2009;45:228-47.

31. Arroyo-Helguera O, Rojas E, Delgado G, Aceves C. Signaling pathways involved in the antiproliferative effect of molecular iodine in normal and tumoral breast cells: evidence that 6-iodolactone mediates apoptotic effects. Endocr Relat Cancer. 2008:15:1003-11.

32. Upadhyay G, Singh R, Sharma R, Balapure AK, Godbole MM. Differential action of iodine on mitochondria from human tumoral- and extratumoral tissue in inducing the release of apoptogenic proteins. Mitochondrion. 2002:2:199-210

33. Rillema JA, Mulder JA. Arachidonic acid distribution in lipids of mammary glands and DMBA-induced tumors of rats. Prostaglandins Med. 1978;1:31-8.

34. Razanamahefa L, Prouff S, Bardon S. Stimulatory effect of arachidonic acid on T-47D human breast cancer cell growth is associated with enhancement of cyclin D1 mRNA expression. Nutr Cancer. 2000;38: 274-80.

35. Aceves C, García-Solís P, Arroyo-Helguera O, Vega-Riveroll L, Delgado G, Anguiano B. Antineoplastic effect of iodine in mammary cancer: participation of 6-iodolactone (6-IL) and peroxisome proliferator-activated receptors (PPAR). Mol Cancer. 2009;8:33-6.

36. Nuñez-Anita RE, Arroyo-Helguera O, Cajero-Juárez M, López-Bojorquez L, Aceves C. A complex between 6-iodolactone and the peroxisome proliferator-activated receptor type gamma may mediate the antineoplastic effect of iodine in mammary cancer. Prostaglandins Other Lipid Mediat. 2009;89:34-42.

37. Reka AK, Kurapati H, Narala VR, Bommer G, Chen J, Standiford TJ, Keshamouni VG. Peroxisome proliferator-activated receptor-gamma activation inhibits tumor metastasis by antagonizing Smad3-mediated epithelial-mesenchymal transition. Mol Cancer Ther. 2010:9:3221-32.

38. Wahl GM, Spike BT. Cell state plasticity, stem cells, EMT, and the generation of intra-tumoral heterogeneity. NPJ Breast Cancer. 2017; https://doi.org/10.1038/s41523-017-0012-z.

39. Asano Y, Kashiwagi S, Goto W, Kurata K, Noda S, Takashima T, Onoda N, Tanaka S, Ohsawa M, Hirakawa K. Tumour-infiltrating CD8 to FOXP3 lymphocyte ratio in predicting treatment responses to neoadjuvant chemotherapy of aggressive breast cancer. Br J Surg. 2016;103:845-54.

40. Denkert C, Loibl S, Noske A, Roller M, Müller BM, Komor M, Budczies J, DarbEsfahani S, Kronenwett R, Hanusch C, von Törne C, Weichert W, Engels K, Solbach C, Schrader I, Dietel M, von Minckwitz G. Tumor-associated lymphocytes as an independent predictor of response to neoadjuvant chemotherapy in breast cancer. J Clinl Oncol. 2010;28:105-13.

41. Beukelman CJ, van den Berg AJ, Hoekstra MJ, Uhl R, Reimer K, Mueller S. Anti-inflammatory properties of a liposomal hydrogel with povidone-iodine (Repithel) for wound healing in vitro. Burns. 2008;34:845-55.

42. Soriguer F, Gutiérrez-Repiso C, Rubio-Martin E, Linares F, Cardona I, López-Ojeda J, Pacheco M, González-Romero S, Garriga MJ, Velasco I, Santiago P, García-Fuentes E. lodine intakes of 100-300 $\mu \mathrm{g} / \mathrm{d}$ do not modify thyroid function and have modest anti-inflammatory effects. Br J Nutr. 2011;105:1783-90

43. Miyachi Y, Niwa Y. Effects of potassium iodide, colchicine and dapsone on the generation of polymorphonuclear leukocyte-derived oxygen intermediates. Br J Dermatol. 1982:209-14.

44. Honma K, Saga K, Onodera H, Takahashi M. Potassium iodide inhibits neutrophil chemotaxis. Acta Derm Venereol. 1990;70:247-9.

45. Costa RO, Macedo PM, Carvalhal A, Bernardes-Engemann AR. Use of potassium iodide in dermatology: updates on an old drug. An Bras Dermatol. 2013:88:396-402.

46. Moore K, Thomas A, Harding KG. lodine released from the wound dressing iodosorb modulates the secretion of cytokines by human macrophages responding to bacterial lipopolysaccharide. Int J Biochem Cell Biol. 1997:29:163-71.

47. McCarty MF, Barroso-Aranda J, Contreras F. PPAR gamma agonists can be expected to potentiate the efficacy of metronomic chemotherapy through CD36 up-regulation. Med Hypotheses. 2008;70:419-23. 
48. Aceves $C$, Anguiano $B$. Is iodine an antioxidant and Antiproliferative agent for the mammary and prostate glands? In: Preedy VR, Burrow GN, Watson RR, editors. Comprehensive Handbook of lodine. Oxford: Academic Press, Elsevier; 2009. p. 249-57.

49. Zava TT, Zava DT. Assessment of Japanese iodine intake based on seaweed consumption in Japan: a literature-based analysis. Thyroid Res. 2011; https://doi.org/10.1186/1756-6614-4-14.

Submit your next manuscript to BioMed Central and we will help you at every step:

- We accept pre-submission inquiries

- Our selector tool helps you to find the most relevant journal

- We provide round the clock customer support

- Convenient online submission

- Thorough peer review

- Inclusion in PubMed and all major indexing services

- Maximum visibility for your research

Submit your manuscript at www.biomedcentral.com/submit
Biomed Central 